\title{
Current progress and mechanisms of bone metastasis in lung cancer: a narrative review
}

\author{
Shengyu $\mathrm{Wu}^{1,2 \#}$, Yue Pan ${ }^{1,2 \#}$, Yanyu Mao ${ }^{1,2 \#}$, Yu Chen ${ }^{3}$, Yayi He ${ }^{1}$ \\ ${ }^{1}$ Department of Medical Oncology, Shanghai Pulmonary Hospital, Tongji University, Shanghai, China; ${ }^{2}$ Medical School, Tongji University, \\ Shanghai, China; ${ }^{3}$ Spine Center, Orthopedic department, Shanghai Changzheng Hospital, Shanghai, China \\ Contributions: (I) Conception and design: Y He, Y Chen; (II) Administrative support: Y He, Y Chen; (III) Provision of study materials or patients: \\ S Wu, Y Pan, Y Mao; (IV) Collection and assembly of data: S Wu, Y Pan, Y Mao; (V) Data analysis and interpretation: S Wu, Y Pan, Y Mao; (VI) \\ Manuscript writing: All authors; (VII) Final approval of manuscript: All authors. \\ \#The authors contributed equally to this work. \\ Correspondence to: Yayi He. Department of Medical Oncology, Shanghai Pulmonary Hospital, Tongji University Medical School Cancer \\ Institute, Tongji University School of Medicine, No. 507 Zhengmin Road, Shanghai 200433, China. Email: 2250601@qq.com; Yu \\ Chen. Spine Center, Orthopedic department, Shanghai Changzheng Hospital, No 415, Fengyang Road, Shanghai 200433 , China. \\ Email: yugangg2003@163.com.
}

\begin{abstract}
Lung cancer is a kind of malignant tumor with rapid progression and poor prognosis. Distant metastasis has been the main cause of mortality among lung cancer patients. Bone is one of the most common sites. Among all lung cancer patients with bone metastasis, most of them are osteolytic metastasis. Some serious clinical consequences like bone pain, pathological fractures, spinal instability, spinal cord compression and hypercalcemia occur as well. Since the severity of bone metastasis in lung cancer, it is undoubtedly necessary to know how lung cancer spread to bone, how can we diagnose it and how can we treat it. Here, we reviewed the process, possible mechanisms, diagnosis methods and current treatment of bone metastasis in lung cancer. We divided the process of bone metastasis in lung cancer into three steps: tumor invasion, tumor cell migration and invasion in bone tissue. It may be influenced by genetic factors, microenvironment and other adhesion-related factors. Imaging examination, laboratory examination, and pathological examination are used to diagnose lung cancer metastasis to bone. Surgery, radiotherapy, targeted therapy, bisphosphonate, radiation therapy and chemotherapy are the common clinical treatment methods currently. We also found some problems remained to be solved. For example, drugs for skeletal related events mainly target on osteoclasts at present, which increase the ratio of patients in osteoporosis and fractures in the long term. In all, this review provides the direction for future research on bone metastasis in lung cancer.
\end{abstract}

Keywords: Lung cancer; bone metastasis; diagnosis methods; mechanism; treatment

Submitted Jul 09, 2020. Accepted for publication Nov 23, 2020.

doi: $10.21037 /$ tlcr-20-835

View this article at: http://dx.doi.org/10.21037/tlcr-20-835

\section{Introduction}

Lung cancer is a kind of malignant tumors with rapid progression and poor prognosis, it brings difficulties to clinical treatment (1). Distant metastases have been the main cause of mortality among lung cancer patients (2). Bone is the second commonest site of lung cancer metastasis (3). According to the characteristics of the lesions, bone metastasis can be divided into the following three types: osteolytic, osteogenic and mixed type (4). Osteolytic bone metastasis account for $70 \%$ and most lung cancer bone metastasis can be classified into this category (5). Bone metastasis can cause pain, pathological fractures, spinal instability, spinal cord compression and hypercalcemia, which impairs the structural integrity of the 
bone, and often predicts a decline in the quality of life and shortened survival of patients (6).

Since the severity of bone metastasis in lung cancer, it is undoubtedly necessary to know how lung cancer spreads to the bone, how can we diagnose it and how can we treat it. We tried to review these from four different aspects: the process of bone metastasis in lung cancer, possible mechanisms, diagnosis methods and current treatment for bone metastasis.

We present the following article in accordance with the Narrative Review reporting checklist (available at http://dx.doi.org/10.21037/tlcr-20-835).

\section{The process of bone metastasis in lung cancer}

The process of bone metastasis in lung cancer could be roughly segmented into three steps: tumor invasion, tumor cell migration and invasion in bone tissue (Figure 1). At first, with the influence of some factors such as protein-protein interactions $(7,8)$ and signaling events $(9,10)$, in particular, the decrease of E-cadherin $(11,12)$ and some proteinases like matrix metalloproteinases (MMPs) (13-15), the status of lung cancer cells are changed. Intercellular adhesion and crosslinking are decreased as well. Thus, tumor cells invade and have a trend of migration. Then, lung cancer cells enter into the circulatory system and are named as circulating tumor cells (CTCs) (16-18). The location of bone metastasis is associated with the red bone marrow (RBM) content (19). The common metastasis sites are spine (20,21), pelvis (19), ribs (22) and so on. This is tumor cell migration. Finally, when the tumor cells reach the surface of the bone, they start to invade. Certain adhesion molecules, for instance, vascular cell adhesion protein-1 (23), play an important role. They promote lung cancer cells joining with the bone cells, beginning to change the bone microenvironment (24).

\section{Types of bone metastasis in lung cancer}

Osteoblast and osteoclast regulate bone changes. They function opposite and maintain relative balance in normal bone shaping and bone homeostasis. The maintenance of bone metabolic balance requires the dynamic balance and coupling between osteoclast-induced bone resorption and osteoblast induced bone formation $(25,26)$. Tumor cells can lead to the imbalance of osteoblasts and osteoclasts, thus interrupting bone reconstruction (27). There are two different types of bone metastasis in lung cancer: osteolytic metastasis and osteogenic metastasis. Among all cases, most lung cancer metastases are osteolytic metastasis caused by osteoclasts.

\section{Osteolytic metastasis}

Evidences showed that lung cancer cells cannot directly cause osteolytic by the secretion of proteolytic enzymes to local tissues (28). Instead, the microenvironment between lung cancer cells and bone tissue played an important role by activating osteoclasts. As a result, osteoclasts destroy the bone and lead to osteolytic lesion (29).

Most studies suggested that osteoblasts were primarily secretory cells that release molecules that regulate osteoclast formation and tumor progression (30). Tumor related factors like bone morphogenetic proteins (BMPs), semaphorin 3A (Sema3A), vascular endothelial growth factor (VEGF), macrophage chemotaxis and activating factor-1 (MCP-1) and interleukin-6 (IL-6) (31-33), have been shown to promote osteoblast differentiation. Besides, miR-139-5p was proved to positively regulate the osteogenic differentiation in mesenchymal stem cells (MSCs) (27). Through the JAK/STAT pathway, leptin can potentiate BMP9-induced osteogenic differentiation of MSCs (34). However, hypoxia inducible factor- $1 \alpha$ (HIF$1 \alpha)$ is engaged in bone metastasis of lung cancer through promoting the expression and secretion of Sema4D to inhibit osteogenic differentiation (35). Besides, dickkopfrelated protein 1 (DKK1) (36) and tumor necrosis factor- $\alpha$ $(\mathrm{TNF}-\alpha)(37)$ have been proved to be involved in inhibiting osteoblast differentiation.

Osteoclasts are the main effector cells in bone resorption. They are derived from hematopoietic progenitor cells in the mononuclear-macrophage family $(38,39)$. Lung cancer cells derived circulating miR-21 promotes the differentiation of monocytes into osteoclasts (40). Myeloidderived suppressor cells (MDSCs), as osteoclast progenitors, can not only differentiate directly into osteoclasts but also produce reactive oxygen species (ROS) and cytokines to inhibit the immune response of host CD4 and CD8 T cells and promote the progress of tumor (41). MiR-34a inhibits osteoclast differentiation and osteoclastic bone metastasis by targeting the inhibition of Tgif2 (42). IL-7 produced by lung cancer cells can up-regulate $T$ cell-derived cytokines such as receptor activator of nuclear factor- $\mathrm{\kappa B}$ ligand (RANKL) and TNF- $\alpha$ to promote osteoclast production (43). Hepatocyte growth factor (HGF)-MET can activate the RANKL/ receptor activator of nuclear factor- $\mathrm{\kappa B}$ (RANK) signaling pathway in the bone microenvironment, induce osteoclasts 


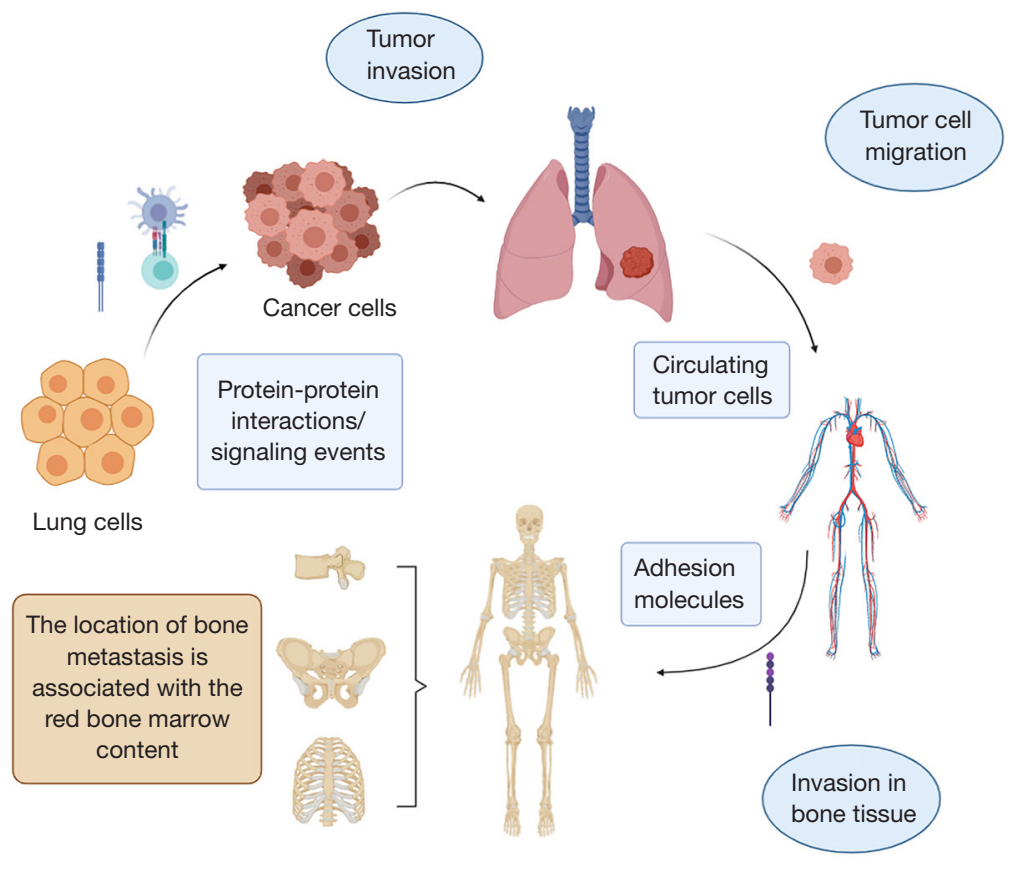

Figure 1 The process of bone metastasis in lung cancer.

activation, and eventually lead to the occurrence of osteolytic metastasis (44).

Osteogenic metastasis bone-resident osteocalcinexpressing $\left(\mathrm{OCN}^{+}\right)$osteoblastic cells are myeloid cells derived from bone marrow. Evidence shows that lung cancer can remotely activate $\mathrm{OCN}^{+}$osteoblasts in bone even in absence of metastasis. Meanwhile, Siglec $\mathrm{F}^{\text {high }}$ neutrophils are provided by these $\mathrm{OCN}^{+}$osteoblasts, thus promoting the development of cancer (45). Therefore, Camilla Engblom and his team revealed that osteoblasts play a remote regulatory role in lung cancer progression but whether osteoblasts are connected with tumor metastasis is pending further discussion.

Compared with osteolytic metastasis, osteoblastic metastasis of lung cancer is rare and if there are unique mechanisms is still unknown. This phenomenon may be linked to some lung cancer-derived factors. For example, $\mathrm{Wnt} / \beta$-catenin signaling and DKK1 cause lung cancer metastasis, especially to bone. However, high expression of DKK1 determines the activity of alkaline phosphatase (ALP) and secretion of osteocalcin, having an impact on differentiation of osteoblast (46).

Overall, the bone metastasis of lung cancer is mostly based on osteolytic metastasis.

\section{Possible mechanisms (Figure 2)}

\section{Genetic factors}

Recently, more and more studies focus on gene regulation to metastasis in lung cancer, especially about miRNA. The miRNA secreted by cancer cells is absorbed by surrounding cells in the form of exosomes and functions. For example, miR-192 overexpressed in lung cancer cells can target vascular endothelial cells through exosomes, inhibit angiogenesis by down-regulating the expression of IL-8, intercellular adhesion molecule (ICAM) and chemokine (C-X-C motif) ligand 1 (CXCL1), thereby reducing the ability of bone metastasis $(47,48)$. It was reported that miRNA-144 targets cyclin E1 (CCNE1) and cyclin E2 (CCNE2) to inhibit non-small cell lung cancer (NSCLC) bone metastases (49). By targeting tripartite motifcontaining 44 (TRIM44), miR-192-5p inhibits lung cancer bone metastasis (50). Exosome miRNAs derived from hypoxic bone marrow mesenchymal stem cells (BMSCs) also evidenced to promote metastasis of lung cancer cells through epithelial-mesenchymal transition (EMT) induced by signal transducers and activators of transcription 3 (STAT3) (51). MiR-335 via insulin-like growth factor-I 


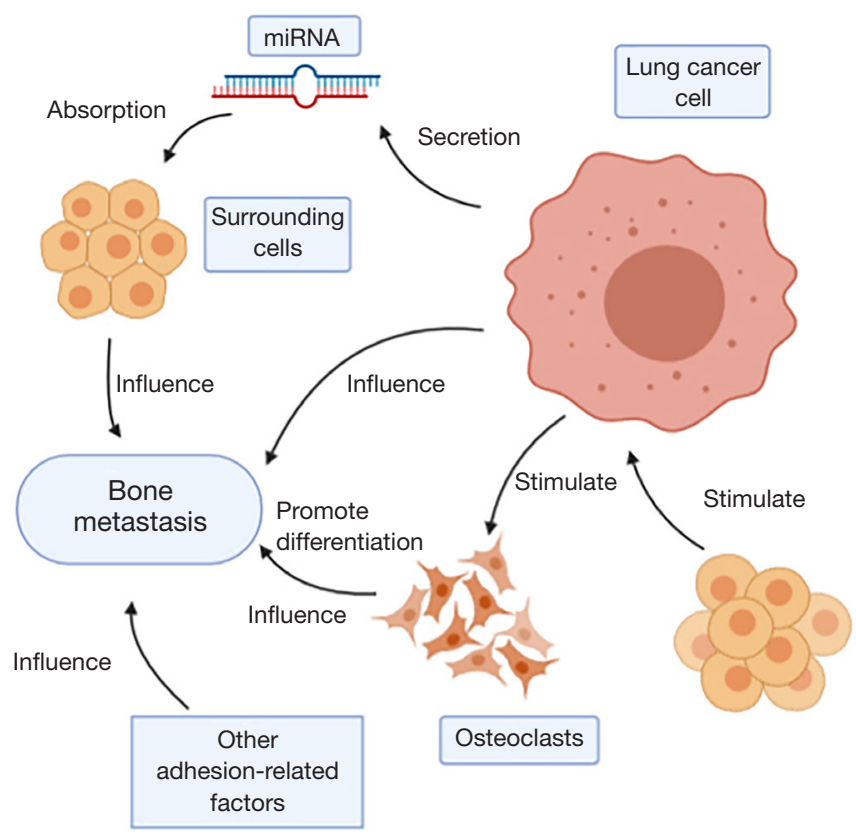

Figure 2 Possible mechanisms.

receptor (IGF-IR) and RANKL pathways inhibited the bone metastases of small cell lung cancer (SCLC) (52). Hsv2-miR-H9-5p is strongly expressed in bone metastasis, which is closely related to the pathology and metastasis of lung cancer (53). Besides, miR-29c is used as a tumor metastasis suppressor, which can directly inhibit the integrin b1 and MMP2 expression, and further reduce the enzyme activity of MMP2, so as to suppress the adhesion of lung cancer cells to extracellular matrix (ECM) and metastasis (54).

Furthermore, DNA is of great significance. For example, by upregulating miR-660-5p and targeted SWItch/Sucrose Non-Fermentable (SWI/SNF) related, matrix associated, actin-dependent regulator of chromatin, subfamily A, member 5 (SMARCA5), Nm23-H1 causes inhibition of bone-specific metastasis (55).

Additionally, mRNA also plays an important role in it. In lung cancer patients, the activation of epidermal growth factor receptor (EGFR) signaling pathway up-regulated angiogenic factors such as VEGF-A and VEGF receptor-1 (VEGFR1) to induce the formation of tumor blood vessels and promote the invasion and metastasis of tumor cells (56). Inversely, down-regulation of $C n A \alpha$ gene expression can reduce the speed of proliferation and colony formation, inhibit the progress of cell cycle, reduce cell migration and invasion, and inhibit cell adhesion to bone matrix (57).

\section{Microenvironment}

Microenvironment plays a fateful role in bone metastasis. Plenty of microenvironmental factors participate in the bone metastasis. BMSCs are guided to tumor cells and help to form a cancer microenvironment (58). Besides, mast cells release tryptase, which can promote tumor cell metastasis through the extracellular body (59). The extracellular CUB2 domain of CUB domain-containing protein 1 (CDCP1) also has a function of promoting cancer cell migration through Src family kinases (SFK) activation on the plasma membrane (60). What's more, the immune cells and natural killer cells (NK cells) in microenvironment work through complex reactions (61). Besides, some mechanisms have been shown to be specific to osteolytic or osteogenic metastasis.

The bone marrow microenvironment plays a key role in osteoclast generation. Leptin can induce cancer cells to secrete soluble ICAMs and induce osteoclast- 
like cell formation (62). Macrophage colony-stimulating factor (M-CSF) secreted by bone marrow stromal cells or osteoblasts can induce osteoclast generation and differentiation (63). Moreover, the RANKL/RANK pathway may be an important link in mediating osteolytic bone destruction in bone metastasis of lung cancer (64). Thromboxane enhances the production of RANKL dependent osteoclasts and promotes bone metastasis of lung cancer (65).

Parathyroid hormone-related protein $(\mathrm{PTHrP})$ is considered to be one of the main regulatory factors in the process of osteolytic bone metastasis in lung cancer, and lung cancer with positive expression is more likely to develop bone metastasis (66). The PTHrP secreted by lung cancer cells binds to the PTH/ PTHrP receptor on the surface of osteoblasts (67), enhancing the expression of RANKL gene on the surface of osteoblasts and decreasing the synthesis of osteoprotegerin (OPG) (68). RANKL binds to RANK on the surface of the precursor cells of osteoclasts, which facilitates the differentiation and maturation of osteoclasts, enhances osteoclasts activities and results in osteolytic bone destruction (69). Repeated intravenous injection of PTHrP neutralizing antibody significantly inhibited the formation of bone metastases in a dose-dependent manner but had no significant effect on metastasis to the internal organs (lung, liver, kidney, lymph nodes) (70). These results suggest that PTHrP is closely related to osteolytic bone metastasis in lung cancer, but has no significant effect on the metastasis of other organs in lung cancer (71).

Growth factors are also a kind of factor contribute to osteolytic metastasis. During osteolytic bone resorption, a large amount of TGF- $\beta$ is released from the bone matrix, which can stimulate disseminated tumor cells (DTCs) to produce more PTHrP, and further up-regulate the expression of RANKL on the surface of bone fine cell and bone basal fine cell, thus exacerbating the formation of osteolytic metastasis (72-74). Inflammatory factor IL-1 $\beta$ may promote the proliferation and migration of lung cancer cells through the COX2-miR-93-Lin28B-let-7 cascade pathway (42).

Furthermore, chemokines also affect bone metastases in the microenvironment. From microarray analysis and validation by a real-time polymerase chain reaction confirmed that $\mathrm{CX} 3 \mathrm{C}$ chemokine ligand 1 (CX3CL1) can be a potential chemokine widely involved in spinal metastases (75). Activation of the KRAS signaling pathway promotes the secretion of chemokine ligand 12 (CCL12) by tumor cells, and the binding of CCL12 to the receptor CXCR4 is conducive to the homing of tumor cells to the target organ (76).

Enzymes participate in the impact of bone metastases as well. Cathepsin A knockout decreases the proliferation and invasion of A549 lung adenocarcinoma cells (77).

\section{Other adhesion-related factors}

Any change of adhesion-related factors may affect the bone metastasis of lung cancer. For example, high expression of lumican in lung cancer cells promotes bone metastasis through the autocrine mechanism (78). The expression of HIF- $1 \alpha$ (79) and LIGHT/TNFSF14 (80) are high in advanced NSCLC. The $\beta 3$-integrin down-regulated cells significantly reduced cell adhesion, migration and invasion (81). Conversely, BMP7 significantly reduces cellular motility of lung cancer cell line, SK-MES1, and its adhesion to Matrigel. RhBMP7 is also able to significantly reduce the invasion of lung cancer cells (82).

\section{Diagnosis methods}

There are many technologies or methods to support the diagnosis. Emission computed tomography (ECT) and positron emission tomography (PET)/computed tomography (CT) are the main methods for screening bone metastases. ECT is currently the screening method of choice for bone metastasis (83). ${ }^{18} \mathrm{~F}$-FDG PET/CT is most sensitive to osteolytic and bone marrow metastasis and can be used as the first choice for patients with bone metastasis of unknown origin (BMUO) $(84,85)$. X-ray, $\mathrm{CT}$ and magnetic resonance imaging (MRI) are common examination methods (86).

Besides all these imaging examinations above, there are some biochemical examinations. The patients with bone metastasis have higher concentrations of cell-free DNA, whose survival outcomes are poorer. So, use of cellfree DNA can provide clinical prognosis management for patients (87). Urinary markers [decrease of circulating tumor DNA (ctDNA) means better overall survival outcome] (88), lactate dehydrogenase values, bone metabolism markers in osteoclasts and osteoblasts, serum level of ALP have the diagnosis significance as well. Apart from that, serological markers like carbohydrate antigen 125 (CA125), carcinoembryonic antigen (CEA), neuron specific enolase (NSE), and squamous cell carcinoma antigen (SCCA) and serum carboxy-terminal telopeptide of 
type I collagen (ICTP) (89) are applied for diagnosis of lung cancer bone metastasis.

The pathological examination is the golden standard (90). In general, imaging examination, laboratory examination, and pathological examination are used to diagnose lung cancer metastasis to bone.

Due to the poor prognosis of lung cancer bone metastasis, early diagnosis of bone metastasis is particularly necessary. Recently, the serological molecular model logit (p) has been proven to have the function of early diagnosis and progression monitoring (91).

\section{Current treatment}

\section{Surgery}

Patients with clinical N0-1 NSCLC with simultaneous bone metastasis may obtain a longer survival rate after primary lung tumor resection. The radically curativeintent surgical resection of the primary lung tumor includes pneumonectomy, systemic decomposition of hilar and mediastinal lymph node dissection $(92,93)$.

\section{Radiotherapy}

Radiotherapy has been shown to improve survival in patients with extensive-stage small cell lung cancer (ESSCLC) with bone metastases, and also significantly decreased the local recurrence rate, especially in patients with only one metastatic site $(94,95)$.

\section{Targeted therapy}

Molecular targeted therapy for lung cancer is a new type of biotherapy model that targets the driving genes that may cause cell canceration and blocks tumor signaling pathways at the molecular level, thereby inhibiting tumor cell growth, inducing apoptosis, and even causing it to completely disappear (5). According to the target of drugs, targeting drugs commonly used in lung cancer are shown in Table 1.

\section{EGFR-tyrosine kinase inhibitor (TKI) inhibitors}

EGFR-TKI can be used as a first-line therapeutic regime option for patients with bone metastases from NSCLC who have a sensitive mutation in the EGFR gene, and can prolong the survival of patients $(96,97)$. Osimertinib is recommended for patients who have progressed after EGFR-TKI treatment and are T790M positive (98).

\section{Phosphoinositol 3 kinase (PI3K) inhibitors: buparlisib} Metastasis of NSCLC results in pathological fracture. Sustained signal transduction by PI3K increases the PI3K dependence and the growth potential of osteoclasts, leading to the continuous growth of NSCLC. Buparlisib, a PI3K inhibitor, inhibited osteoclast formation presenting antitumor activity, so that targeting PI3K pathway may be a potential therapeutic strategy (99).

\section{Statins as inhibitors: fluvastatin}

It is known that long-term use of statins can reduce the risk of various cancers. Statins can directly inhibit osteoclastogenesis and the proliferation of cancer cells by blocking the post-translational modifications of RhoA and Ras (100). Statins such as fluvastatin have been reported to inhibit tumor progression and greatly reduce the ability of tumor cells to migrate and invade. In addition, fluvastatin induces $\mathrm{p} 53$ which activate autophagy of cancer cells. The induction of autophagy can exert an anti-cancer effect in an immune-dependent or independent manner (101).

\section{Denosumab}

Denosumab is a monoclonal antibody against RANK ligand specifically, which can significantly improve the survival rate without bone metastasis, and has the best therapeutic effect on advanced high-risk population (102). Denosumab does not require kidney monitoring or dose adjustment, because of the convenience of it with subcutaneous drug delivery $(103,104)$.

\section{Cabozantinib}

Cabozantinib is a multi-target, small-molecule inhibitor of MET and VEGFR2 that can be taken orally (105). Cabozantinib's clinical activity is manifested in the introduction of bone cell blockage and osteolytic lesions into the process to reduce soft tissue lesions, improve progression-free survival (PFS), reduce the resolution of bone scan and reduce bone turnover markers (106).

\section{Latest progress}

The matricellular glycoprotein thrombospondin (TSP-2) promotes osteoclastogenesis through RANKL pathway and changes the RANKL:OPG ratio in differentiation of osteoblasts. It has been proved that TSP-2 gene knockout inhibits osteolytic metastasis of lung cancer in vivo so it may be the possible target for treatment in the future (65).

Ribosomal protein lateral stalk subunit P2 (RPLP2), enolase 1 (ENO1) and NME/NM23 nucleoside diphosphate 
Table 1 Targeting drugs commonly used in lung cancer

\begin{tabular}{|c|c|c|}
\hline Experimental drugs & Year & Experiment name \\
\hline PI3K inhibitors: buparlisib & 2019 & The effects of a PI3K inhibitor on NSCLC growth in bone and osteoclast formation \\
\hline Statins as inhibitors: fluvastatin & 2017 & $\begin{array}{l}\text { p53 in both fluvastatin-induced autophagy and suppression of lung adenocarcinoma bone } \\
\text { metastasis }\end{array}$ \\
\hline Cabozantinib & 2013 & $\begin{array}{l}\text { Cabozantinib in patients with advanced prostate cancer: results of a phase II randomized } \\
\text { discontinuation trial }\end{array}$ \\
\hline
\end{tabular}

EGFR, epidermal growth factor receptor; TKI, tyrosine kinase inhibitor.

kinase 2 (NME1-NME2) express high in bone metastasis patients with poor prognosis. They are also identified as therapeutic targets for bone metastasis in lung cancer (107).

Tumor-microenvironment in lung cancer-metastasis also works and provides some drug targets. For instance, tests in rat models found that reduced L1CAM-expressionto has relations with the lower rate of bone metastasis. This represents a potentially targetable regulating factor.

\section{Bisphosphonate}

Some bisphosphonates have direct anti-tumor effects and can be used in combination with conventional anti-tumor therapy to treat bone metastases from lung cancer, which can reduce skeletal related events (SREs), improve pain control, and show a trend of increased survival $(5,108)$. Nitrogen-containing bisphosphonates can delay the growth of bone tumors by inhibiting bone destruction mediated by osteoclasts and preventing the release of cytokines and growth factors that destroy bones. It can also inhibit the function of tumor cells by inhibiting farnesyl diphosphate synthases (FPPS), such as the adhesion, migration, invasion, and proliferation of tumor cells and the adhesion of bone matrix, thus induces apoptosis of tumor cells (109). One of the most well-known bisphosphonate drugs is zoledronic acid. Its treatment is well tolerated, significantly reducing the incidence of bone-related events and increasing the time for the first occurrence of bone-related events (110).

\section{Radiation therapy}

Radiation therapy is one of the effective methods to treat bone metastasis of lung cancer, including external radiation therapy and radionuclide therapy. Through this treatment, a series of symptoms can be alleviated or eliminated, pathologic fractures can be alleviated, spinal cord compression can be reduced, quality of life can be improved, and survival period can be extended (5).

\section{Chemotherapy}

Chemotherapy is widely used in the therapy of bone metastases in lung cancer, where cancer drugs are used to ease the pain. But the general anti-tumor chemotherapeutic drugs have some side effects, such as toxic reaction, bone marrow suppression and so on. Therefore, anti-tumor chemotherapeutic drugs should be carefully selected in the clinical application (111). For example, paclitaxel and kanglaite are common anticancer drugs in cancer chemotherapy. Paclitaxel has reliable anticancer activity. Kanglaite, an effective component extracted from semen coicis, can inhibit cancer cell growth and improve the patient's immune capacity. Paclitaxel and kanglaite are commonly used in chemotherapy for bone metastasis of lung cancer. In accordance with research, paclitaxel combined with kanglaite is more effective than paclitaxel or kanglaite alone in improving bone metastasis of lung cancer, which is of great help in the clinical treatment (111).

In summary, surgery, radiotherapy, targeted therapy, bisphosphonate and chemotherapy are the current treatment methods. They can be used alone or in combination.

\section{Methods}

When it comes to research selection, literature search was applied in PubMed (112). MedLine, PreMedline and other electronic publications were included. Considering the timeliness of the literature, most of the cited references are 
within 3 years. They were all English literature. We chose "lung cancer", "bone metastasis" and so on as keywords to search for the appropriate references for this review.

\section{Summary}

After tumor invasion, tumor cell migration and invasion in bone tissue, lung cancer finishes the process of bone metastasis. This process is a combined action of genetic factors, microenvironment and other adhesion related factors. We can diagnose it by many technologies, mainly imaging examinations, biochemical examinations and pathological examination, which is the golden standard. Surgery, radiotherapy, targeted therapy and bisphosphonate are the current treatment.

Because the review quoted the latest literature, we may have neglected to describe the results of earlier studies on bone metastases in lung cancer. Moreover, our review may not be the whole literature collection. But it is still a detailed review from the aspect of process, mechanism, diagnosis methods and current treatment in lung cancer metastasis.

However, lots of issues remain the focus of future research. Bone metastasis of lung cancer involves a wide range of mechanisms and any change in any step may accelerate tumor metastasis. What's more, the osteoblastic metastasis of lung cancer is rare, so whether it exists remains to be confirmed. SRE is also a problem related to tumor metastasis. Though we choose bisphosphonate to prevent it, its value in fracture prevention is uncertain at present. Other drugs like denosumab, a monoclonal antibody targeting RANKL (113), also have side effects on bone destruction like medication-related osteonecrosis of the jaw (MRONJ) (114). Enzalutamide was proved to significantly delay the first-time SREs in patients with metastatic castrated prostate cancer who received chemotherapy (115) but the effect has not been confirmed in lung cancer yet. Evidence shows that radiotherapy combined with zoledronate can reduce SREs in renal cell carcinoma (116). Drugs for SREs mentioned above mainly target on osteoclasts, increasing the probability of osteoporosis and fractures. Other treatments have no good therapeutic effect on fracture as well. Fractures caused by drugs for SREs in lung cancer is one of the problems remain to be solved in bone metastasis of lung cancer. This is also what our team will focus on in the future.

Therefore, this review summarized the current situation in bone metastasis of lung cancer to provide new direction for future treatment.

\section{Acknowledgments}

Funding: This study was supported in part by a grant of Young Talents in Shanghai, National Natural Science Foundation of China (81802255), Young Talents in Shanghai (2019 QNBJ), 'Dream Tutor' Outstanding Young Talents Program (fkyq1901), Clinical Research Project of Shanghai Pulmonary Hospital (fk18005), Key Discipline in 2019 (oncology), Project of Shanghai Municipal Science and Technology Commission (Project of Municipal Science and Technology Commission), Scientific Research Project of Shanghai Pulmonary Hospital (fkcx1903), Shanghai Municipal Commission of Health and Family Planning (2017YQ050), Innovation Training Project of SITP of Tongji University, and Key Projects of Leading Talent (19411950300). Youth Project of Hospital Management Research Fund of Shanghai Hospital Association (Q1902037).

\section{Footnote}

Reporting Checklist: The authors have completed the Narrative Review reporting checklist. Available at http://dx.doi.org/10.21037/tlcr-20-835

Conflicts of Interest: All authors have completed the ICMJE uniform disclosure form (available at http://dx.doi.org/10.21037/tlcr-20-835). The authors have no conflicts of interest to declare.

Ethical Statement: The authors are accountable for all aspects of the work in ensuring that questions related to the accuracy or integrity of any part of the work are appropriately investigated and resolved. This research was approved by the Ethics Committee of the Shanghai Pulmonary Hospital, Tongji University.

Open Access Statement: This is an Open Access article distributed in accordance with the Creative Commons Attribution-NonCommercial-NoDerivs 4.0 International License (CC BY-NC-ND 4.0), which permits the noncommercial replication and distribution of the article with the strict proviso that no changes or edits are made and the original work is properly cited (including links to both the formal publication through the relevant DOI and the license). See: https://creativecommons.org/licenses/by-nc-nd/4.0/. 


\section{References}

1. The L. Lung cancer: some progress, but still a lot more to do. Lancet 2019;394:1880.

2. Altorki NK, Markowitz GJ, Gao D, et al. The lung microenvironment: an important regulator of tumour growth and metastasis. Nat Rev Cancer 2019;19:9-31.

3. Akoury E, Ramirez Garcia Luna AS, Ahangar P, et al. Anti-Tumor Effects of Low Dose Zoledronate on Lung Cancer-Induced Spine Metastasis. J Clin Med 2019;8.

4. Fornetti J, Welm AL, Stewart SA. Understanding the Bone in Cancer Metastasis. J Bone Miner Res 2018; 33:2099-113.

5. Zheng XQ, Huang JF, Lin JL, et al. Incidence, prognostic factors, and a nomogram of lung cancer with bone metastasis at initial diagnosis: a population-based study. Transl Lung Cancer Res 2019;8:367-79.

6. Zhang L, Gong Z. Clinical Characteristics and Prognostic Factors in Bone Metastases from Lung Cancer. Med Sci Monit 2017;23:4087-94.

7. Friedl P, Wolf K. Tumour-cell invasion and migration: diversity and escape mechanisms. Nat Rev Cancer 2003;3:362-74.

8. Lauffenburger DA, Horwitz AF. Cell migration: a physically integrated molecular process. Cell 1996; 84:359-69.

9. Hu T, Lu YR. BCYRN1, a c-MYC-activated long noncoding RNA, regulates cell metastasis of non-small-cell lung cancer. Cancer Cell Int 2015;15:36.

10. Burridge K, Chrzanowska-Wodnicka M. Focal adhesions, contractility, and signaling. Annu Rev Cell Dev Biol 1996;12:463-518.

11. Perl AK, Wilgenbus P, Dahl U, et al. A causal role for E-cadherin in the transition from adenoma to carcinoma. Nature 1998;392:190-3.

12. Yan B, Zhang W, Jiang LY, et al. Reduced E-Cadherin expression is a prognostic biomarker of non-small cell lung cancer: a meta-analysis based on 2395 subjects. Int J Clin Exp Med 2014;7:4352-6.

13. Hsu CP, Shen GH, Ko JL. Matrix metalloproteinase-13 expression is associated with bone marrow microinvolvement and prognosis in non-small cell lung cancer. Lung Cancer 2006;52:349-57.

14. Coussens LM, Fingleton B, Matrisian LM. Matrix metalloproteinase inhibitors and cancer: trials and tribulations. Science 2002;295:2387-92.

15. Wojnacki J, Quassollo G, Marzolo MP, et al. Rho GTPases at the crossroad of signaling networks in mammals: impact of Rho-GTPases on microtubule organization and dynamics. Small GTPases 2014;5:e28430.

16. O'Flaherty JD, Gray S, Richard D, et al. Circulating tumour cells, their role in metastasis and their clinical utility in lung cancer. Lung Cancer 2012;76:19-25.

17. Parkinson DR, Dracopoli N, Petty BG, et al. Considerations in the development of circulating tumor cell technology for clinical use. J Transl Med 2012;10:138.

18. Cheng M, Liu L, Yang HS, et al. Circulating tumor cells are associated with bone metastasis of lung cancer. Asian Pac J Cancer Prev 2014;15:6369-74.

19. Onken JS, Fekonja LS, Wehowsky R, et al. Metastatic dissemination patterns of different primary tumors to the spine and other bones. Clin Exp Metastasis 2019;36:493-8.

20. Kelly PD, Zuckerman SL, Than KD, et al. Metastatic spine disease in lung cancer patients: national patterns of radiation and surgical care. J Spine Surg 2019;5:320-8.

21. Gong L, Xu L, Yuan Z, et al. Clinical outcome for small cell lung cancer patients with bone metastases at the time of diagnosis. J Bone Oncol 2019;19:100265.

22. Gu H, Sun L, Dou Z, et al. Analysis of lung adenocarcinoma with bone metastasis: a case report. Transl Lung Cancer Res 2020;9:389-92.

23. Yoneda T, Hiraga T. Crosstalk between cancer cells and bone microenvironment in bone metastasis. Biochem Biophys Res Commun 2005;328:679-87.

24. Luo Q, Xu Z, Wang L, et al. Progress in the research on the mechanism of bone metastasis in lung cancer. Mol Clin Oncol 2016;5:227-35.

25. Chen X, Wang Z, Duan N, et al. Osteoblast-osteoclast interactions. Connect Tissue Res 2018;59:99-107.

26. Kikuta J, Ishii M. Bone Imaging: Osteoclast and Osteoblast Dynamics. Methods Mol Biol 2018;1763:1-9.

27. Xu S, Yang F, Liu R, et al. Serum microRNA-139-5p is downregulated in lung cancer patients with lytic bone metastasis. Oncol Rep 2018;39:2376-84.

28. Yin JJ, Pollock CB, Kelly K. Mechanisms of cancer metastasis to the bone. Cell Res 2005;15:57-62.

29. Sela J. Bone remodeling in pathologic conditions. A scanning electron microscopic study. Calcif Tissue Res 1977;23:229-34.

30. Han Y, You X, Xing W, et al. Paracrine and endocrine actions of bone-the functions of secretory proteins from osteoblasts, osteocytes, and osteoclasts. Bone Res 2018;6:16.

31. Rahim F, Hajizamani S, Mortaz E, et al. Molecular regulation of bone marrow metastasis in prostate and breast cancer. Bone Marrow Res 2014;2014:405920. 
32. Liu F, Shen W, Qiu H, et al. Prostate cancer cells induce osteoblastic differentiation via semaphorin 3A. Prostate 2015;75:370-80.

33. Jeong HM, Cho SW, Park SI. Osteoblasts Are the Centerpiece of the Metastatic Bone Microenvironment. Endocrinol Metab (Seoul) 2016;31:485-92.

34. Zhang B, Yang L, Zeng Z, et al. Leptin Potentiates BMP9Induced Osteogenic Differentiation of Mesenchymal Stem Cells Through the Activation of JAK/STAT Signaling. Stem Cells Dev 2020;29:498-510.

35. Chen WG, Sun J, Shen WW, et al. Sema4D expression and secretion are increased by HIF-1alpha and inhibit osteogenesis in bone metastases of lung cancer. Clin Exp Metastasis 2019;36:39-56.

36. Chu T, Teng J, Jiang L, et al. Lung cancer-derived Dickkopf1 is associated with bone metastasis and the mechanism involves the inhibition of osteoblast differentiation. Biochem Biophys Res Commun 2014;443:962-8.

37. Wang LM, Zhao N, Zhang J, et al. Tumor necrosis factoralpha inhibits osteogenic differentiation of pre-osteoblasts by downregulation of EphB4 signaling via activated nuclear factor-kappaB signaling pathway. J Periodontal Res 2018;53:66-72.

38. Heymann D, Guicheux J, Gouin F, et al. Cytokines, growth factors and osteoclasts. Cytokine 1998;10:155-68.

39. Kiyoi T. Bone Resorption Activity in Mature Osteoclasts. Methods Mol Biol 2018;1868:215-22.

40. Zhao Q, Liu C, Xie Y, et al. Lung Cancer Cells Derived Circulating miR-21 Promotes Differentiation of Monocytes into Osteoclasts. Onco Targets Ther 2020;13:2643-56.

41. Roato I. Bone metastases: When and how lung cancer interacts with bone. World J Clin Oncol 2014;5:149-55.

42. Wang L, Zhang LF, Wu J, et al. IL-1beta-mediated repression of microRNA-101 is crucial for inflammationpromoted lung tumorigenesis. Cancer Res 2014; 74:4720-30.

43. Roato I, Caldo D, Godio L, et al. Bone invading NSCLC cells produce IL-7: mice model and human histologic data. BMC Cancer 2010;10:12.

44. Lath DL, Buckle CH, Evans HR, et al. ARQ-197, a smallmolecule inhibitor of c-Met, reduces tumour burden and prevents myeloma-induced bone disease in vivo. PLoS One 2018;13:e0199517.

45. Engblom C, Pfirschke C, Zilionis R, et al. Osteoblasts remotely supply lung tumors with cancer-promoting SiglecFhigh neutrophils. Science 2017;358:eaal5081.
46. Zhuang X, Zhang H, Li X, et al. Differential effects on lung and bone metastasis of breast cancer by Wnt signalling inhibitor DKK1. Nat Cell Biol 2017;19:1274-85.

47. Ell B, Kang Y. MicroRNAs as regulators of bone homeostasis and bone metastasis. Bonekey Rep 2014;3:549.

48. Valencia K, Luis-Ravelo D, Bovy N, et al. miRNA cargo within exosome-like vesicle transfer influences metastatic bone colonization. Mol Oncol 2014;8:689-703.

49. Liang Y, Zhang D, Li L, et al. Exosomal microRNA-144 from bone marrow-derived mesenchymal stem cells inhibits the progression of non-small cell lung cancer by targeting CCNE1 and CCNE2. Stem Cell Res Ther 2020;11:87.

50. Zou P, Zhu M, Lian C, et al. miR-192-5p suppresses the progression of lung cancer bone metastasis by targeting TRIM44. Sci Rep 2019;9:19619.

51. Zhang X, Sai B, Wang F, et al. Hypoxic BMSC-derived exosomal miRNAs promote metastasis of lung cancer cells via STAT3-induced EMT. Mol Cancer 2019;18:40.

52. Gong M, Ma J, Guillemette R, et al. miR-335 inhibits small cell lung cancer bone metastases via IGF-IR and RANKL pathways. Mol Cancer Res 2014;12:101-10.

53. Wang X, Liu S, Zhou Z, et al. A herpes simplex virus type 2-encoded microRNA promotes tumor cell metastasis by targeting suppressor of cytokine signaling 2 in lung cancer. Tumour Biol 2017;39:1010428317701633.

54. Wang H, Zhu Y, Zhao M, et al. miRNA-29c suppresses lung cancer cell adhesion to extracellular matrix and metastasis by targeting integrin beta 1 and matrix metalloproteinase2 (MMP2). PLoS One 2013;8:e70192.

55. Ai C, Ma G, Deng Y, et al. Nm23-H1 inhibits lung cancer bone-specific metastasis by upregulating miR-660-5p targeted SMARCA5. Thorac Cancer 2020;11:640-50.

56. Larsen AK, Ouaret D, El Ouadrani K, et al. Targeting EGFR and VEGF(R) pathway cross-talk in tumor survival and angiogenesis. Pharmacol Ther 2011;131:80-90.

57. Ma NQ, Liu LL, Min J, et al. The effect of down regulation of calcineurin Aalpha by lentiviral vectormediated RNAi on the biological behavior of small-cell lung cancer and its bone metastasis. Clin Exp Metastasis 2011;28:765-78.

58. Sai B, Dai Y, Fan S, et al. Cancer-educated mesenchymal stem cells promote the survival of cancer cells at primary and distant metastatic sites via the expansion of bone marrow-derived-PMN-MDSCs. Cell Death Dis 2019;10:941.

59. Xiao H, He M, Xie G, et al. The release of tryptase from 
mast cells promote tumor cell metastasis via exosomes. BMC Cancer 2019;19:1015.

60. Sawayama T, Nakashima K, Ichimura T, et al. Homophilic complex formation of CDCP1 via the extracellular CUB2 domain facilitates SFK activation and promotes cancer cell migration. Oncol Rep 2019. [Epub ahead of print]. doi: 10.3892/or.2019.7271.

61. Roato I, Vitale M. The Uncovered Role of Immune Cells and NK Cells in the Regulation of Bone Metastasis. Front Endocrinol (Lausanne) 2019;10:145.

62. Tsai CF, Chen JH, Wu CT, et al. Induction of osteoclastlike cell formation by leptin-induced soluble intercellular adhesion molecule secreted from cancer cells. Ther Adv Med Oncol 2019;11:1758835919846806.

63. Ruef N, Dolder S, Aeberli D, et al. Granulocytemacrophage colony-stimulating factor-dependent CD11cpositive cells differentiate into active osteoclasts. Bone 2017;97:267-77.

64. Feeley BT, Liu NQ, Conduah AH, et al. Mixed metastatic lung cancer lesions in bone are inhibited by noggin overexpression and Rank:Fc administration. J Bone Miner Res 2006;21:1571-80.

65. Wang M, Chao CC, Chen PC, et al. Thrombospondin enhances RANKL-dependent osteoclastogenesis and facilitates lung cancer bone metastasis. Biochem Pharmacol 2019;166:23-32.

66. Muguruma H, Yano S, Kakiuchi S, et al. Reveromycin A inhibits osteolytic bone metastasis of small-cell lung cancer cells, SBC-5, through an antiosteoclastic activity. Clin Cancer Res 2005;11:8822-8.

67. Kir S, Komaba H, Garcia AP, et al. PTH/PTHrP Receptor Mediates Cachexia in Models of Kidney Failure and Cancer. Cell Metab 2016;23:315-23.

68. D'Oronzo S, Brown J, Coleman R. The role of biomarkers in the management of bone-homing malignancies. J Bone Oncol 2017;9:1-9.

69. Dou C, Li J, Kang F, et al. Dual Effect of Cyanidin on RANKL-Induced Differentiation and Fusion of Osteoclasts. J Cell Physiol 2016;231:558-67.

70. Fritsch $\mathrm{S}$, Lindner $\mathrm{V}, \mathrm{Welsch} \mathrm{S}$, et al. Intravenous delivery of PTH/PTHrP type 1 receptor cDNA to rats decreases heart rate, blood pressure, renal tone, renin angiotensin system, and stress-induced cardiovascular responses. J Am Soc Nephrol 2004;15:2588-600.

71. Miki T, Yano S, Hanibuchi M, et al. Parathyroid hormonerelated protein (PTHrP) is responsible for production of bone metastasis, but not visceral metastasis, by human small cell lung cancer SBC-5 cells in natural killer cell- depleted SCID mice. Int J Cancer 2004;108:511-5.

72. Kim J, Bae JS. Tumor-Associated Macrophages and Neutrophils in Tumor Microenvironment. Mediators Inflamm 2016;2016:6058147.

73. Farajzadeh Valilou S, Keshavarz-Fathi M, Silvestris N, et al. The role of inflammatory cytokines and tumor associated macrophages (TAMs) in microenvironment of pancreatic cancer. Cytokine Growth Factor Rev 2018;39:46-61.

74. Yahaya MAF, Lila MAM, Ismail S, et al. TumourAssociated Macrophages (TAMs) in Colon Cancer and How to Reeducate Them. J Immunol Res 2019;2019:2368249.

75. Liu W, Bian C, Liang Y, et al. CX3CL1: a potential chemokine widely involved in the process spinal metastases. Oncotarget 2017;8:15213-9.

76. Renaud S, Falcoz PE, Schaeffer M, et al. Prognostic value of the KRAS G12V mutation in 841 surgically resected Caucasian lung adenocarcinoma cases. Br J Cancer 2015;113:1206-15.

77. Hu B, Zhu X, Lu J. Cathepsin A knockdown decreases the proliferation and invasion of A549 lung adenocarcinoma cells. Mol Med Rep 2020;21:2553-9.

78. Hsiao KC, Chu PY, Chang GC, et al. Elevated Expression of Lumican in Lung Cancer Cells Promotes Bone Metastasis through an Autocrine Regulatory Mechanism. Cancers (Basel) 2020;12:233.

79. Pezzuto A, Perrone G, Orlando N, et al. A close relationship between $\mathrm{HIF}$-1alpha expression and bone metastases in advanced NSCLC, a retrospective analysis. Oncotarget 2019;10:7071-9.

80. Brunetti G, Belisario DC, Bortolotti S, et al. LIGHT/ TNFSF14 Promotes Osteolytic Bone Metastases in Nonsmall Cell Lung Cancer Patients. J Bone Miner Res 2020;35:671-80.

81. Li N, Zhang JP, Guo S, et al. Down-regulation of beta3integrin inhibits bone metastasis of small cell lung cancer. Mol Biol Rep 2012;39:3029-35.

82. Zhao Q, Li P, Ma J, et al. MicroRNAs in Lung Cancer and Lung Cancer Bone Metastases: Biomarkers for Early Diagnosis and Targets for Treatment. Recent Pat Anticancer Drug Discov 2015;10:182-200.

83. Ichinose $\mathrm{Y}$, Hara N, Ohta M, et al. Preoperative examination to detect distant metastasis is not advocated for asymptomatic patients with stages 1 and 2 non-small cell lung cancer. Preoperative examination for lung cancer. Chest 1989;96:1104-9.

84. D'Addario G, Felip E, Group EGW. Non-small-cell 
lung cancer: ESMO clinical recommendations for diagnosis, treatment and follow-up. Ann Oncol 2009;20 Suppl 4:68-70.

85. Budak E, Yanarates A. Role of (18)F-FDG PET/CT in the detection of primary malignancy in patients with bone metastasis of unknown origin. Rev Esp Med Nucl Imagen Mol 2020;39:14-9.

86. Mazzone PJ, Silvestri GA, Patel S, et al. Screening for Lung Cancer: CHEST Guideline and Expert Panel Report. Chest 2018;153:954-85.

87. Ye Y, Luo Z, Shi D. Use of cell free DNA as a prognostic biomarker in non-small cell lung cancer patients with bone metastasis. Int J Biol Markers 2019;34:381-8.

88. Jin PC, Gou B, Qian W. Urinary markers in treatment monitoring of lung cancer patients with bone metastasis. Int J Biol Markers 2019;34:243-50.

89. Horiguchi T, Tachikawa S, Kondo R, et al. Usefulness of serum carboxy-terminal telopeptide of type I collagen (ICTP) as a marker of bone metastasis from lung cancer. Jpn J Clin Oncol 2000;30:174-9.

90. Schwartz AM, Rezaei MK. Diagnostic surgical pathology in lung cancer: Diagnosis and management of lung cancer, 3rd ed: American College of Chest Physicians evidence-based clinical practice guidelines. Chest 2013;143:e251S-e262S.

91. Teng X, Wei L, Han L, et al. Establishment of a serological molecular model for the early diagnosis and progression monitoring of bone metastasis in lung cancer. BMC Cancer 2020;20:562.

92. Takahashi Y, Adachi H, Mizukami Y, et al. Patient outcomes post-pulmonary resection for synchronous bone-metastatic non-small cell lung cancer. J Thorac Dis 2019;11:3836-45.

93. Nakazawa S, Shimizu K, Nagashima T, et al. Quality of life after video-assisted surgery for lung cancer. Lancet Oncol 2016;17:e318.

94. Shang X, Lin J, Li Z, et al. Radiotherapy may improve survival of ES-SCLC with distant metastasis only for patients with one metastatic site: A population-based study. Oncol Lett 2020;19:139-46.

95. Rades D, Haus R, Janssen S, et al. An easy-to-use scoring system to estimate the survival of patients irradiated for bone metastases from lung cancer. Transl Lung Cancer Res 2020;9:1067-73.

96. Wang D, Luo Y, Shen D, et al. Clinical features and treatment of patients with lung adenocarcinoma with bone marrow metastasis. Tumori 2019;105:388-93.

97. Sugiura H, Yamada K, Sugiura T, et al. Predictors of survival in patients with bone metastasis of lung cancer. Clin Orthop Relat Res 2008;466:729-36.

98. Tsai PC, Yeh YC, Huang CS, et al. Pathological complete response after afatinib treatment of stage IV oligometastatic adenocarcinoma of the lung: the role of pulmonary surgery. Surg Case Rep 2019;5:178.

99. Wang S, Niu X, Bao X, et al. The PI3K inhibitor buparlisib suppresses osteoclast formation and tumour cell growth in bone metastasis of lung cancer, as evidenced by multimodality molecular imaging. Oncol Rep 2019;41:2636-46.

100. Sarkar D. Statins as Inhibitors of Lung Cancer Bone Metastasis. EBioMedicine 2017;19:6-7.

101. Yang Z, Su Z, DeWitt JP, et al. Fluvastatin Prevents Lung Adenocarcinoma Bone Metastasis by Triggering Autophagy. EBioMedicine 2017;19:49-59.

102.Smith MR, Saad F, Coleman R, et al. Denosumab and bone-metastasis-free survival in men with castrationresistant prostate cancer: results of a phase 3, randomised, placebo-controlled trial. Lancet 2012;379:39-46.

103. Henry DH, Costa L, Goldwasser F, et al. Randomized, double-blind study of denosumab versus zoledronic acid in the treatment of bone metastases in patients with advanced cancer (excluding breast and prostate cancer) or multiple myeloma. J Clin Oncol 2011;29:1125-32.

104. Scagliotti GV, Hirsh V, Siena S, et al. Overall survival improvement in patients with lung cancer and bone metastases treated with denosumab versus zoledronic acid: subgroup analysis from a randomized phase 3 study. J Thorac Oncol 2012;7:1823-9.

105. Smith MR, Sweeney CJ, Corn PG, et al. Cabozantinib in chemotherapy-pretreated metastatic castration-resistant prostate cancer: results of a phase II nonrandomized expansion study. J Clin Oncol 2014;32:3391-9.

106. Smith DC, Smith MR, Sweeney C, et al. Cabozantinib in patients with advanced prostate cancer: results of a phase II randomized discontinuation trial. J Clin Oncol 2013;31:412-9.

107. Yang M, Sun Y, Sun J, et al. Differentially expressed and survival-related proteins of lung adenocarcinoma with bone metastasis. Cancer Med 2018;7:1081-92.

108.Lopez-Olivo MA, Shah NA, Pratt G, et al. Bisphosphonates in the treatment of patients with lung cancer and metastatic bone disease: a systematic review and meta-analysis. Support Care Cancer 2012;20:2985-98.

109. Coleman RE, Lipton A, Roodman GD, et al. Metastasis and bone loss: advancing treatment and prevention. Cancer Treat Rev 2010;36:615-20. 
110. Rosen LS. Efficacy and safety of zoledronic acid in the treatment of bone metastases associated with lung cancer and other solid tumors. Semin Oncol 2002;29:28-32.

111. Cao L, Long L, Hu C. Efficacy of Paclitaxel Combined with Kanglaite Injection in Treatment of Bone Metastases of Lung Cancer. Iran J Public Health 2019;48:1445-51.

112. Fiorini N, Lipman DJ, Lu Z. Towards PubMed 2.0. Elife 2017;6:e28801.

113. Raje N, Terpos E, Willenbacher W, et al. Denosumab versus zoledronic acid in bone disease treatment of newly diagnosed multiple myeloma: an international, doubleblind, double-dummy, randomised, controlled, phase 3 study. Lancet Oncol 2018;19:370-81.

114. Otto S, Pautke C, Van den Wyngaert T, et al. Medication- related osteonecrosis of the jaw: Prevention, diagnosis and management in patients with cancer and bone metastases. Cancer Treat Rev 2018;69:177-87.

115.Loriot Y, Miller K, Sternberg CN, et al. Effect of enzalutamide on health-related quality of life, pain, and skeletal-related events in asymptomatic and minimally symptomatic, chemotherapy-naive patients with metastatic castration-resistant prostate cancer (PREVAIL): results from a randomised, phase 3 trial. Lancet Oncol 2015;16:509-21.

116. Hosaka S, Katagiri H, Niwakawa M, et al. Radiotherapy combined with zoledronate can reduce skeletal-related events in renal cell carcinoma patients with bone metastasis. Int J Clin Oncol 2018;23:1127-33.

Cite this article as: Wu S, Pan Y, Mao Y, ChenY, He Y. Current progress and mechanisms of bone metastasis in lung cancer: a narrative review. Transl Lung Cancer Res 2021;10(1):439-451. doi: 10.21037/tlcr-20-835 